\title{
Integrating Irinotecan in standard chemotherapy: a novel dose density combination for High-Risk pediatric Sarcomas
}

\author{
Gianni Bisogno ${ }^{1}$, Andrea Ferrari ${ }^{2}$, Arianna Tagarelli ${ }^{1}$, Silvia Sorbara ${ }^{1}$, Stefano Chiaravalli ${ }^{2}$, \\ Elena Poli ${ }^{1}$, Giovanni Scarzello ${ }^{3}$, Federica De Corti ${ }^{1}$, Michela Casanova ${ }^{2}$, and Maria \\ Carmen Affinita ${ }^{1}$ \\ ${ }^{1}$ Padua University Hospital \\ ${ }^{2}$ Fondazione IRCCS Istituto Nazionale dei Tumori \\ ${ }^{3}$ Istituto Oncologico Veneto Istituto di Ricovero e Cura a Carattere Scientifico
}

October 20, 2020

\begin{abstract}
BACKGROUND: Irinotecan is a drug active against pediatric sarcomas with a toxicity profile that theoretically allows for its association with more myelotoxic drugs. We examined the feasibility of a dose-density strategy integrating irinotecan in standard chemotherapy regimens for patients with high-risk sarcomas. METHODS: Between November 2013 and January 2020 , 23 patients $<21$ years old with metastatic (11 children) or recurrent (12 children) sarcomas were treated with 9 IrIVA/IrVAC cycles. All newly-diagnosed patients received IrIVA (ifosfamide $3 \mathrm{~g} / \mathrm{m} 2$ on days 1 and 2, vincristine $1.5 \mathrm{mg} / \mathrm{m} 2 \mathrm{on}$ day 1 , actinomycin D $1.5 \mathrm{mg} / \mathrm{m} 2$ on day 1 , irinotecan $20 \mathrm{mg} / \mathrm{m} 2$ for 5 consecutive days starting on day 8). Two relapsed patients received IrIVA and 10 IrVAC (cyclophosphamide $1.5 \mathrm{~g} / \mathrm{m} 2$ on day 1 instead of ifosfamide). Feasibility was assessed in terms of toxicity and time to complete the treatment. RESULTS: 17 rhabdomyosarcomas, 4 Ewing sarcomas, 2 desmoplastic round cell tumors received a total of 181 cycles (range 2-10). Grade 4 neutropenia occurred in $62.4 \%$ of the cycles. 13 patients had febrile neutropenia. Diarrhea occurred in 14 cycles. The median time to complete the treatment was 195 days (range 170-231), $83.4 \%$ of cycles were administered on time or with a delay $<1$ week. With a median follow-up of 2.6 years (range 0.2-5.0), 12 patients are alive, 9 complete remissions, 3 with the disease. Conclusions: A dose density strategy combining irinotecan with standard chemotherapy is feasible. This approach will be investigated in the next trial coordinated by the European pediatric Soft tissue sarcoma Study Group.
\end{abstract}

\section{INTRODUCTION}

Sarcomas represent a heterogeneous group of rare tumors with a variety of histological and biological characteristics and different prognosis. In pediatric age, rhabdomyosarcoma (RMS) is the most common histotype. Like Ewing sarcoma (ES) and desmoplastic small round cell tumors (DSRCT), RMS is a high-grade tumor with a strong tendency for local invasiveness and metastatic spread. These tumors are currently treated using a multidisciplinary approach that includes surgery, radiotherapy, and intensive multi-agent chemotherapy. While the outcome of treatment for patients with localized RMS and ES is generally a 70-80\% survival rate, the outcome remains unsatisfactory for patients with distant metastases and those who relapse (1) (2). Patients with DSRCT still carry a poor prognosis in most cases (3) and, together with those who have metastatic or relapsed RMS and ES, form a high-risk group for whom new treatment approaches need to be investigated.

The use of new drugs to target specific molecular alterations is an attractive approach for poor prognosis sarcomas. However, at this time, the activity data of these new agents is still limited (4) (5). An improvement in the outcome of children with high-risk sarcomas may also be pursued by optimizing the use of already- 
available drugs known to be active against this disease. The treatment of pediatric sarcomas is currently based on administering chemotherapy cycles containing multiple drugs every three weeks. It may be that cancer cells start growing again during the interval between chemotherapy cycles and develop a resistance to the drugs administered.

Reducing the time between chemotherapy cycles to intensify the treatment has proved feasible and effective in the treatment of ES (6) and, to some degree, for metastatic RMS (7). Higher treatment intensity can also be achieved by increasing the dosage of drugs per unit of time, according to the so-called dose-density approach.

Irinotecan is active against various pediatric tumors, including RMS and other sarcomas (8). As it is relatively non-myelotoxic, we hypothesized that it might be associated with other more myelotoxic drugs.

In this study, we explored the feasibility of a dose-density strategy for patients with high-risk soft-tissue sarcomas that involved a novel combination of irinotecan with a more standard regimen based on alkylating agents. This represents a proof of concept that will be further investigated in the next multicenter trial coordinated by the European pediatric Soft tissue sarcoma Study Group (EpSSG).

\section{METHOD}

This study included patients with a histologically confirmed diagnosis of RMS, ES, or DSRCT with metastasis at diagnosis or with relapsed/refractory disease. To be eligible, patients had to be $>6$ months and [?] 21 years old, and have: a Karnofsky performance status of 70-100\% (for patients $>12$ years old) or a Lansky Play Score of $70-100 \%$ (for patients [?]12 years of age); an adequate bone marrow function (absolute neutrophil

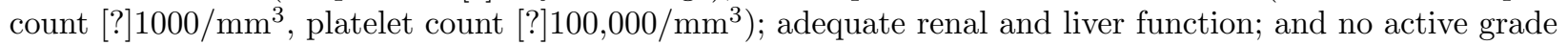
$>2$ diarrhea or uncontrolled infection.

The study was approved by the local ethical committee, and informed consent was obtained from patients or parents, as appropriate.

The diagnostic workup consisted of CT and/or MRI scans of the primary tumor, chest CT scan, radionuclide bone scan, bone marrow aspirate, and biopsy. 18F-fluorodeoxyglucose PET was optional.

\section{TREATMENT}

As detailed in Figure 1, the first part of the regimen investigated consisted of IVA ( ifosfamide at a dose of $3 \mathrm{~g} / \mathrm{m}^{2}$ on Days 1 and 2 as a 3-hour intravenous infusion (iv) (with sodium mercaptoethanesulfonate at a dose of $3 \mathrm{~g} / \mathrm{m}^{2}$ per day and hyperhydration); vincristine at a dose of $1.5 \mathrm{mg} / \mathrm{m}^{2}$ ( maximum, $2 \mathrm{mg}$ ) on Day 1 given as a single bolus dose by intravenous injection; actinomycin at a dose of $1.5 \mathrm{mg} / \mathrm{m}^{2}$ (maximum, $2 \mathrm{mg}$ ) on Day 1 given as a single bolus by intravenous injection, VAC (vincristine $1.5 \mathrm{mg} / \mathrm{m}^{2}$ iv, actinomycin D $1.5 \mathrm{mg} / \mathrm{m}^{2}$ iv, and cyclophosphamide $1.5 \mathrm{mg} / \mathrm{m}^{2}$, all on day 1) currently used respectively by the EpSSG in Europe, and by the Children's Oncology Group (COG) in the USA. VAC was administered to patients that received ifosfamide in the first line. Irinotecan was started on day 8 and given for 5 days (preferably Monday to Friday to enable its administration in the outpatient setting), irrespective of blood cell counts, providing the patient was in good clinical conditions. Since this was the first attempt to include irinotecan in a dose-density multidrug combination, a fixed, reduced dose of irinotecan was used $\left(20 \mathrm{mg} / \mathrm{m}^{2} /\right.$ day for 5 consecutive days) to gather information and ascertain whether a subsequent formal phase I trial would be possible. Diarrhea prophylaxis included the administration of cefixime $8 \mathrm{mg} / \mathrm{kg}$ per os once a day for 9 consecutive days starting on day 6 .

Cycles were administered every 21 days, with neutrophils $>1.0 \times 10^{9} / 1$ and platelets to $>100 \times 10^{9} / 1$ and following resolution of non-hematopoietic toxicity. The preventive use of colony-stimulating factors was not allowed. Tumor response was scheduled to be assessed after 3 and 9 cycles at the site of both the primary tumor and any metastases, and more assessments were possible if clinically indicated. The response was classified as: complete response $(\mathrm{CR})=$ complete disappearance of all visible disease; partial response $(\mathrm{PR})$ $=$ a tumor volume reduction of more than two-thirds; minor response $(\mathrm{MR})=$ a tumor volume reduction of 
more than one-third, but less than two-thirds. A reduction in volume of less than one-third was recorded as stable disease (SD), while an increase in tumor size or the detection of new lesions was classified as progression of disease $(\mathrm{PD})$

Local treatment with radiotherapy and/or surgery had to be considered after the $4^{\text {th }}$ cycle of chemotherapy, but only general recommendations were given because of the heterogeneity of the study population. Resection was recommended for primary tumors with/without metastases, if feasible. Radiotherapy also had to be considered for primary and metastatic sites in newly-diagnosed metastatic sarcomas. If possible, re-irradiation was recommended in relapsed patients.

Toxicity monitoring and recommendations for changes to chemotherapy

During chemotherapy, complete blood cell counts and blood chemistry were obtained at the baseline, weekly, and before each cycle. Toxicity was graded using the National Cancer Institute's Common Toxicity Criteria (CTC), version 5.0. In the event of significant diarrhea ([?] CTC grade III), the dose of irinotecan had to be reduced by $25 \%$ for the subsequent course. If there were further episodes, the dose had to be further reduced or even omitted.

Drug doses for younger patients had to be calculated by body surface area, then reduced by $50 \%$ or $33 \%$ for children under 6 or 12 months old, respectively.

As a general criterion for evaluating the feasibility of this new regimen, we established that more than $70 \%$ of cycles would have to be administered with a delay of less than one week. We also compared the overall duration of the treatment with a historical series of patients with metastatic RMS treated in Italy from November 2013 to January 2020 and included in the EpSSG MTS-2008 protocol. These patients received 9 cycles of chemotherapy at 3-week intervals: 4 cycles of the IVADo regimen (ifosfamide, vincristine, actinomycin D, and doxorubicin) followed by 5 cycles of IVA (ifosfamide, vincristine, actinomycin D) (9). These patients subsequently received maintenance therapy with cyclophosphamide and vinorelbine, but this part of the treatment was not considered for the purposes of our comparison.

\section{RESULTS}

Between November 2013 to January 2020, this study enrolled 23 patients aged from 1.3 to 25 years

(median 10.9 years), and 15 were male. The study population included 11 newly-diagnosed metastatic sarcomas (10 RMS and 1 DSRCT) and 12 relapsed tumors (7 RMS, 4 ES, and 1 DSRCT). All patients with metastatic disease had important metastatic diffusion. The patients with DSRCT had peritoneal dissemination (Table 1).

All newly-diagnosed patients received the IrIVA combination, while in the group of relapsed patients, 2 received IrIVA and 10 IrVAC.

\section{Toxicity}

In total, 181 cycles (range 2-10) were administered, and 173 were evaluable for toxicity (105 IrIVA and 68 IrVAC cycles). The treatment was stopped in two patients after 2 and 7 cycles due to tumor progression, and in another two after 3 and 5 cycles due to the clinician's or parents' decision. Another patient was given high-dose chemotherapy.

No toxic deaths occurred. As expected, the most significant toxicity was myelosuppression. Grade 4 neutropenia was observed in 108 cycles $(62.4 \%)$ and was more frequent in patients receiving $\operatorname{IrVAC}(72 \%$ of cycles) than in those given IrIVA (56.1\%). Grade 3 anemia was evident in 40 cycles $(23.1 \%)$, with 14 patients requiring blood transfusions (no grade 4 anemia was reported). Thrombocytopenia was less severe, with grade 4 toxicity observed in only 7 cycles $(4.0 \%)$, and 4 patients receiving platelet transfusions (Table1S-2S-3S). There were 33 episodes of fever during neutropenia (20.2\% of cycles) in 13 patients, and blood cultures were positive in 4 cases, but without any signs of septic shock. One patient had an episode of typhlitis, and one had surgery for appendicitis. 
Six patients experienced 19 episodes of grade 3 non-hematological toxicity (Table 2); no grade 4 episodes were recorded.. Diarrhea occurred in 14 cycles, with 3 episodes of grade 3 diarrhea in two patients, one of them experienced two episodes during febrile neutropenia (in one case a stool test was positive for Clostridium difficilis ). The dose of irinotecan was not reduced in any subsequent cycles because the patients recovered rapidly. No meaningful difference in toxicity emerged between patients given IrIVA or IrVAC.

Constipation and peripheral neuropathy prompted a reduction in the dose of vincristine in 15 cycles. The irinotecan cycles were delayed for more than one week in 7 cycles, and not administered due to fever and neutropenia in 2 cycles. The dose of irinotecan was never reduced. Ifosfamide was replaced with cyclophosphamide in a child suffering from seizures. Actinomycin was withdrawn during radiotherapy in 7 cycles.

The median interval between cycles was 23 days (range 19-51), with 151 out of 181 cycles (83.4\%) administered on time or without any significant delay ( $<1$ week). Considering the patients who completed the whole chemotherapy treatment (i.e., excluding the 4 patients that prematurely concluded the treatment), the median interval between the first and ninth cycles was 195 days (range 170-231). On comparing this outcome with the 40 patients enrolled in the MTS2008 protocol, we found that precisely the same median time - 195 days (range 169-270) - was taken to complete 9 cycles of chemotherapy.

Nine patients had a delay of more than 30 days, mainly due to local therapies.

\section{Outcome}

After the initial three cycles, CR was evident in 3 patients, PR in 15, and MR in 1 . SD was evident in the 2 children with RMS and PD in 2 patients with Ewing sarcoma. Considering the CR and PR alone, the response rate was $88.2 \%(15 / 17)$ in cases of RMS. PR was evident in $2 / 4$ patients with relapsed ES and $1 / 2$ patients with DSRCT. The response rates were $84.6 \%(11 / 13)$ and $70 \%(7 / 10)$ for patients treated with IrIVA and IrVAC, respectively.

With a median follow-up of 2.6 years (range $0.2-5.0$ ), 12 patients were still alive at the time of writing, 9 in CR (3 metastatic at diagnosis and 6 with the relapsing disease) and 3 were alive with disease.

\section{DISCUSSION}

In this study, we evaluated the feasibility of a dose density approach using a chemotherapy combination that aimed to include irinotecan in the chemotherapy regimens currently used to treat patients with sarcoma, and especially RMS.

The IVA regimen is now considered the standard chemotherapy in European trials for non-metastatic RMS. In North America, cyclophosphamide is used instead of ifosfamide in the VAC regimen. The most significant toxicity of these two combinations is myelotoxicity in the 2-3 weeks following their administration. Irinotecan is a drug active against RMS and, when administered with a continuous schedule (i.e., over 5 days for 1 week), its worst side effect is diarrhea, while myelotoxicity is low.

The IrIVA or IrVAC regimens are, therefore, rational combinations of the standard IVA/VAC scheme with irinotecan because the activity of the drugs and their different toxicity profiles theoretically allow an increase of the chemotherapy intensity without increasing the risk of toxicity.

This was confirmed in our study. Most of the patients were able to complete their treatment within a reasonable time. Although IrIVA/IrVAC are intensive combinations, toxicity was not significantly worse than in patients treated with IVA in the RMS2005 protocol, where grade 3-4 infections were reported in $57 \%$ of patients, and renal and gastrointestinal toxicity in $2 \%$ and $8 \%$, respectively (10). It should be noted that $47.8 \%$ of the patients in our present study had a relapsed tumor, so they had already received previous treatments, meaning that a more significant toxicity might have been expected.

There is limited experience of the dose-density approach against pediatric sarcoma. An attempt to reduce the interval between cycles proved feasible, and possibly beneficial, in patients with metastatic RMS included in the COG protocol ARST0431study was based on the concept of dose-compression: the cycles of chemotherapy 
were administered with an interval of 14 days instead of the 21 as it is used in the standard treatment. The results showed a possible improvement for patients with embryonal RMS, but not for those with high-risk features (Oberlin score >1) (7). More promising results were achieved in localized ES by adopting the same chemotherapy-intensifying approach and reducing the interval between cycles to 2 weeks. A randomized study reported a significantly better outcome in patients treated with chemotherapy administered after shorter intervals than in those given the same chemotherapy according to the standard interval (6). New combinations are under study using the same approach (11).

In our high-risk population, $52 \%$ of patients were alive in $\mathrm{CR}$ at the time of writing, although some have only a short follow-up. This result, and the limited toxicity reported, indicate that the IrIVA or IrVAC combinations deserve further testing, possibly in a more homogenous group of patients with less unfavorable features-as already done for ES patients (6) (11).

A limitation of our study is that we tested only one dose of irinotecan $\left(20 \mathrm{mg} / \mathrm{m}^{2} /\right.$ day for 5 days $)$. The recommended dose of irinotecan, when it is used alone or with vincristine, is $50 \mathrm{mg} / \mathrm{m}^{2}$ for 5 days. Therefore a proper phase I study is needed to establish the maximum tolerated dose of irinotecan within this novel regime.

In conclusion, our study demonstrates that it is feasible to include irinotecan in the standard chemotherapy regimens used to treat sarcoma, and its use in this setting deserves further investigation. This will be one of the goals of the next EpSSG study.

\section{Conflict of interest statement}

The authors have no conflicts of interest to disclose.

\section{Acknowledgments}

We would like to thank Angela Scagnellato and Beatrice Coppadoro for their help with data management and analysis.

This work was supported by the Fondazione Lucia Valentini Terrani ONLUS

\section{REFERENCES}

1. Oberlin, O.; Rey, A.; Lyden, E.; et al. Prognostic factors in metastatic rhabdomyosarcomas: Results of a pooled analysis from United States and European Cooperative Groups. J Clin Oncol. 2008 May 10;26(14):2384-9. 2. Compostella, A.; Affinita, M.C.; Casanova, M.; et al. Topotecan/carboplatin regimen for refractory/recurrent rhabdomyosarcoma in children: Report from the AIEOP Soft Tissue Sarcoma Committee. Tumori. 2019 Apr;105(2):138-143. 3. Honoré, C.; Delhorme, J.B.; Nassif, E. et al. Can we cure patients with abdominal Desmoplastic Small Round Cell Tumor? Results of a retrospective multicentric study on 100 patients. Surg Oncol 2019 Jun;29:107-112. 4. Chisholm, J.C.; Merks, J.H.M.; Casanova, M.;et al. Open-label, multicentre, randomised, phase II study of the EpSSG and the ITCC evaluating the addition of bevacizumab to chemotherapy in childhood and adolescent patients with metastatic soft tissue sarcoma (the BERNIE study). Eur J Cancer. 2017 Sep;83:177-184. 5. Jin, W. The Role of Tyrosine Kinases as a Critical Prognostic Parameter and Its Targeted Therapies in Ewing Sarcoma. Front Cell Dev Biol. 2020 Jul 9;8:613. 6. Womer, R.B.; West, D.C.; Krailo, M.D.; et al. Randomized controlled trial of interval-compressed chemotherapy for the treatment of localized ewing sarcoma: A report from the children's oncology group. J Clin Oncol. 2012 Nov 20;30(33):4148-54 7. Weigel, B.J.; Lyden, E.; Anderson, J.R.; et al. Intensive multiagent therapy, including dose-compressed cycles of ifosfamide/etoposide and vincristine/doxorubicin/cyclophosphamide, irinotecan, and radiation, in patientswith high-risk rhabdomyosarcoma: A report from the children's oncology group. J Clin Oncol. 2016 Jan 10;34(2):117-22. 8. Wagner, L.M. Fifteen years of irinotecan therapy for pediatric sarcoma: where to next? Clin Sarcoma Res. 2015 Aug 28;5:20 9. Bisogno, G.; Ferrari, A.; Bergeron, C.; et al. The IVADo regimen - A pilot study with ifosfamide, vincristine, actinomycin D, and doxorubicin in children with metastatic soft tissue sarcoma: A pilot study on behalf of the European pediatric soft tissue sarcoma study group. Cancer. 2005 Apr 15;103(8):1719-24. 
10. Bisogno, G.; Jenney, M.; Bergeron, C.; et al. Addition of dose-intensified doxorubicin to standard chemotherapy for rhabdomyosarcoma (EpSSG RMS 2005): a multicentre, open-label, randomised controlled, phase 3 trial Lancet Oncol. 2018 Aug;19(8):1061-1071. 11. Mascarenhas, L.; Felgenhauer, J.L.; Bond, M.C.; et al. Pilot Study of Adding Vincristine, Topotecan, and Cyclophosphamide to Interval-Compressed Chemotherapy in Newly Diagnosed Patients With Localized Ewing Sarcoma: A Report From the Children's Oncology Group. Pediatr Blood Cancer. 2016 Mar;63(3):493-8

\section{Table and figure legends}

Table 1 - Patients' characteristics

Table 2: Non-hematological toxicity

Figure 1. The IrIVA regimen

\section{Supporting Information}

Table 1S: Hematologic toxicity -Whole population (173 cycles)

Table 2S: Hematologic toxicity -Patients treated with IrIVA (Total number of cycles : 105)

Table3S: Hematologic toxicity -Patients treated with IrVAC(Total number of cycles: 68)

\section{Hosted file}

Table 1 \selectlanguage\{english\}-- Patients\selectlanguage\{english\}' characteristics.pdf available at https://authorea.com/users/338247/articles/487603-integrating-irinotecan-instandard-chemotherapy-a-novel-dose-density-combination-for-high-risk-pediatric-sarcomas

\section{Hosted file}

Table 2 - Non-hematological toxicity.pdf available at https://authorea.com/users/338247/ articles/487603-integrating-irinotecan-in-standard-chemotherapy-a-novel-dose-densitycombination-for-high-risk-pediatric-sarcomas 
Figure 1.The IrIVA regimen.

\begin{tabular}{|c|c|c|c|c|c|c|c|c|}
\hline $\begin{array}{lll} & & \\
V & \mathrm{~V} & \mathrm{~V} \\
\mathrm{~A} & \mathrm{Ir} & \end{array}$ & $\begin{array}{lll}\mathrm{I} & & \\
\mathrm{V} & \mathrm{V} & \mathrm{V} \\
\mathrm{A} & \mathrm{Ir}\end{array}$ & $\begin{array}{lll}I & & \\
V & V & V \\
A & I r & \end{array}$ & $\begin{array}{ll}I & \\
V & V \\
A & I r\end{array}$ & $\begin{array}{ll} & \\
\text { V } & \text { V } \\
A & \text { Ir }\end{array}$ & $\begin{array}{ll}\mathrm{I} & \\
\mathrm{V} & \mathrm{V} \\
\mathrm{A} & \mathrm{Ir}\end{array}$ & $\begin{array}{ll}I & \\
\text { V } & \text { V } \\
A & \text { Ir }\end{array}$ & 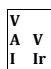 & $\begin{array}{ll}I & \\
\text { V } & \text { V } \\
A & \text { Ir }\end{array}$ \\
\hline
\end{tabular}

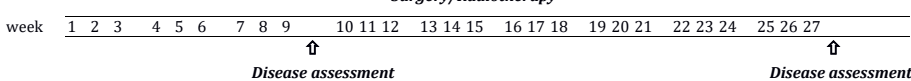

Legend: I: ifosfamide- $3 \mathrm{~g} / \mathrm{m} 2$ is given as a 3-hour intravenousinfusion (iv) daily with sodium mercaptoethanesulfonate $\left(3 \mathrm{~g} / \mathrm{m}^{2}\right)$ and hydration on
Days 1 and 2 of each course of treatment (total dose per course $\left.6 \mathrm{~g} / \mathrm{m}^{2}\right): \mathrm{V}$ : vincristine- $1.5 \mathrm{mg} / \mathrm{m}^{2}($ maximum single dose $2 \mathrm{mg}$ ) is given as a single iv on Days 1 and 8 of each course (and on Day 15 of each of the initial 3 courses): A actinomycin D $-1.5 \mathrm{mg} / \mathrm{m}^{2}$ (maximum single dose $2 \mathrm{mg}$ ) is iv on Days 1 and 8 of each course (and on Day 15 of each of the initial 3 courses); A: actinomycin D - $1.5 \mathrm{mg} / \mathrm{m}^{2}$ (maximum single dose $2 \mathrm{mg}$ ) is
givenas a single iv on Day 1 of each course; irinotecan at a dose of $20 \mathrm{mg} / \mathrm{m}^{2}$ is given as a 30 -minute infusion for 5 days starting on day 8 of each
course. 